\title{
Vielfältiges Engagement
}

\section{Philipp Bertschinger ${ }^{a}$, \\ Ursula Zybach ${ }^{b}$, \\ Peter Bauerfeind, Urs Marbet ${ }^{d}$}

\footnotetext{
a Dr. med., Präsident SGG-SSG

b Leiterin Präventionsprogramme, Krebsliga Schweiz

c Prof. Dr. med., Präsident MDLS

d Prof. Dr. med.
} Chefarzt Kantonsspital Uri

Korrespondenz:

Dr. med. Philipp Bertschinger Präsident SGG-SSG GastroZentrum Hirslanden Witellikerstrasse 40 CH-8032 Zürich Tel. 0443873955 philipp.bertschinger[at] gastrozentrum.ch
Kollege Binswanger publiziert regelmässig teilweise provokativ formulierte Thesen in dieser Rubrik, die teils durchaus den Finger auf wichtige Punkte setzen.

$\mathrm{Zu}$ Recht stellt Herr Kollege Binwanger fest, dass das Screening nach kolorektalen Karzinomen eine Bedeutung erlangt hat, die nicht nur einer Elite vorbehalten sein sollte. Das Kolo-Rektal-Karzinom (KRK) ist in den westlichen Ländern häufig (zweit- bis dritthäufigstes Karzinom bei Männern und Frauen). Ohne Screening wird die Diagnose bei über 70\% der Patienten erst in fortgeschrittenem Stadium gestellt. Daher erstaunt es überhaupt nicht, dass am Tumorboard immer wieder fortgeschrittene Karzinome vorgestellt werden.

Die Schweizerische Gesellschaft für Gastroenterologie SGG hat früh die Problematik in Bezug auf das Screening des kolorektalen Karzinoms erkannt und deshalb ein entsprechendes Papier (Kolon-KarzinomeScreening in der Schweiz - Die Position der SGG-SSG) dem BAG zugestellt mit dem Antrag, die Thematik zu prüfen und ein Screening für nicht risikobelastete Personen zuzulassen. Gerade weil die SGG ein opportunistisches, gewisse Gruppen bevorzugendes Screening schon zu Beginn als problematisch erachtete, hat sie Position für ein systematisches Screening bezogen. Leider ist das Projekt 2006 von den Behörden nicht weiter verfolgt worden.

Ebenfalls seit langer Zeit engagieren sich die Krebsliga Schweiz und ihre Regionalgruppen mit verschiedenen Projekten, um die Prognose des kolorektalen Karzinoms in der Schweiz zu verbessern. Dieses Jahr gelang es ihr, mitgetragen vom Kollegium Hausarztmedizin, der Schweizerischen Gesellschaft für Gastroenterologie SGG-SSG, der Magendarmliga Schweiz, der pharmaSuisse, der Schweizerischen Gesellschaft für Viszeralchirurgie und dem Schweizerischen Verband der Brustkrebsfrüherkennungsprogramme nach langer Vorarbeit ein äusserst umfangreiches Dossier mit einem Antrag auf Kostenübernahme des Darmkrebsscreenings durch die obligatorische Krankenpflegeversicherung im Rahmen eines Kolon Karzinom Programmes (KKP) an das BAG zuhanden der Eidgenössischen Leistungs- und Grundsatzkommission (ELGK) und dem Eidgenössischen Departement des Innern einzureichen. Das Dossier beleuchtet sehr ausführlich wissenschaftliche, gesundheitspolitische und ökonomische Aspekte eines solchen Programms in der Schweiz. Die dabei durchgeführten ökonomischen Analysen kamen sogar zum Schluss, dass ein solches Programm beinahe kostenneutral sein könnte, und dass die Ressourcen in unserem Lande durchaus genügen könnten. Anzumerken ist in diesem Zusammenhang, dass die ScreeningKoloskopie z. B. in Deutschland als qualitativ hochwertiger und schwieriger als die «normale» Koloskopie eingestuft wird. Gerade für diese Untersuchung macht es daher keinen Sinn, weniger gut ausgebildetes Personal einzusetzen. Gutausgebildete Gastroenterologen haben nachgewiesenermassen eine höhere Entdeckungsrate von Karzinomen und Polypen. Ausserdem wäre es wesentlich teurer, wenn Untersuchte, bei denen Polypen gefunden werden, eine zweite Koloskopie zur Polypektomie bei einem «erfahrenen» Gastroenterologen durchführen müssten.

Kollege Binswanger hat recht, die zitierte Antwort des Spezialisten zeugt von Zynismus, aber noch mehr von Unkenntnis. Es gibt kaum ein Karzinom, dessen Entstehung wir derart gut kennen, wie jene des Darmkrebses, weshalb es in der Theorie seit langem klar ist, dass es Wege gibt, wie die Prognose dieses Karzinoms verbessert werden könnte. In einer renommiert publizierten randomisierten, kontrollierten Studie konnte gezeigt werden, dass mittels Rectosigmoidoskopie in diesem Bereich das Karzinom teilweise verhindert und früher erkannt werden konnte. Endlich konnte hiermit auch mit einer randomisierten, kontrollierten Studie bewiesen werden, dass das endoskopische Screening nützt. Wie Richard O. Binswanger richtig festhält, entdeckt das endoskopische Screening das Karzinom nicht nur früher, sondern es verhindert es oft.

$\mathrm{Zu}$ Recht betont Herr Kollege Binswanger zudem, dass ein Pilotprojekt durchgeführt werden sollte. Seit dem Jahre 2000 läuft in einer Region der Schweiz ein Pilotprojekt, das teilweise bereits publiziert ist (Marbet UA. Endoscopy; 2008). Über 70\% der Karzinome wurden in einem Frühstadium entdeckt, was ohne Screening nur bei $20 \%$ der Fall war. Die prospektive Langzeitkontrolle zeigte zudem, dass die Inzidenz und noch mehr die tumorbedingte Mortalität massiv abnahm (eingereicht zur Veröffentlichung). Auch der Kanton Waadt ist nach einer ersten Machbarkeitsstudie nun in der Evaluationsphase für ein Pilotprojekt «DarmkrebsScreening» innerhalb des Kantons.

Herr Kollege Binswanger, wir sind froh, dass Sie ein wichtiges Thema aufgreifen, und wir hoffen, dass die Bedeutung erkannt wird, damit endlich auch in der Schweiz ein sinnvolles ethisch vertretbares Screeningprogramm zur Verhütung des kolorektalen Karzinoms eingeführt wird. 